\title{
Proximity-Induced Superconductivity in a Topological Crystalline Insulator
}

Bryan Rachmilowitz ${ }^{1,}{ }^{*}$, He Zhao ${ }^{1,}{ }^{*}$, Hong Li ${ }^{1}$, Alex LaFleur ${ }^{1}$, J. Schneeloch ${ }^{2}$, Ruidan Zhong ${ }^{2}$, Genda $\mathrm{Gu}^{2}$ and Ilija Zeljkovic ${ }^{1,9}$

${ }^{1}$ Department of Physics, Boston College, 140 Commonwealth Ave, Chestnut Hill, MA 02467; ${ }^{2}$ Brookhaven National Laboratory, Upton, NY 11973; " Corresponding author: ilija.zeljkovic@bc.edu; "Equal contribution.

\begin{abstract}
.
Superconducting topological crystalline insulators (TCl) are predicted to host new topological phases protected by crystalline symmetries, but available materials are insufficiently suitable for surface studies. To induce superconductivity at the surface of a prototypical $\mathrm{TCl} \mathrm{SnTe}$, we use molecular beam epitaxy to grow a heterostructure of SnTe and a high- $T_{c}$ superconductor Fe(Te,Se), utilizing a "buffer" layer to bridge the large lattice mismatch between SnTe and Fe(Te,Se). Using low-temperature scanning tunneling microscopy and spectroscopy, we measure a prominent spectral gap on the surface of SnTe, and demonstrate its superconducting origin by its dependence on temperature and magnetic field. Our work provides a new platform for atomic-scale investigations of emergent topological phenomena in superconducting $\mathrm{TCls}$.
\end{abstract}

\section{Introduction.}

Topological crystalline insulators (TCls) are a subclass of topological materials in which the emergence of non-trivial surface states is intimately tied to a discrete set of crystalline symmetries 1. Numerous types of symmetries can in principle lead to a $\mathrm{TCl}$ phase ${ }^{1}$, but only one class of $\mathrm{TCls}$ based on the reflection symmetry with respect to the (110) mirror plane ${ }^{2}$ has so far been experimentally realized in rock-salt $(\mathrm{Pb}, \mathrm{Sn}) \mathrm{Se}$ and $(\mathrm{Pb}, \mathrm{Sn}) \mathrm{Te}^{3-6}$. The electronic band structure of these TCls consists of multiple Dirac fermions, tunable by temperature ${ }^{3,6}$, chemical composition ${ }^{7-}$ ${ }^{9}$ and various types of strain ${ }^{9-12}$, and provides a rich playground for uncovering new physics ${ }^{13-17}$. For example, a single unit cell thick film of SnTe is shown to exhibit room-temperature ferroelectricity ${ }^{14}$, while a bulk single crystal of $(\mathrm{Pb}, \mathrm{Sn}) \mathrm{Se}$ is reported to host $1 \mathrm{D}$ edge modes propagating along step edges ${ }^{15,17}$. Theory predicts that if a $\mathrm{TCl}$ undergoes a superconducting 
transition, a new topological superconducting phase could emerge ${ }^{18-21}$ distinct from that in proximitized $Z_{2}$ topological insulators $\mathrm{Bi}_{2}(\mathrm{Se}, \mathrm{Te})_{3}{ }^{22-28}$. The difference is rooted in the unique mirror symmetry protection and multiple Dirac fermions present at the surface of TCls, which are expected to facilitate novel phenomena emerging at the surface. These include multiple symmetry-protected Majorana zero modes inside a single vortex core ${ }^{20}$ and tunable Andreev bound states ${ }^{29}$. The bottleneck in exploring these lies in the synthesis of high-quality surfaces of superconducting TCls.

In analogy to achieving superconductivity in $Z_{2}$ topological insulators ${ }^{30}$, alloying has been successfully used to induce bulk superconductivity in TCls ${ }^{31}$. Bulk single crystals of $S_{1-x} \ln _{\mathrm{x}} T e$ exhibit superconductivity up to $\sim 4.5 \mathrm{~K}^{31}$ and maintain the topological nature of the Dirac surface states ${ }^{32-34}$. However, the difficulty in obtaining a large, flat surface of $\mathrm{Sn}_{1-\mathrm{x}} \ln \mathrm{x}$ Te by cleaving has hindered nanoscale explorations of the superconducting $\mathrm{TCl}$ phase. An alternative method to induce superconductivity in a material, without introducing chemical disorder, could entail using the proximity effect ${ }^{35}$. This approach typically involves deposition or exfoliation of thin films on top of superconducting substrates, and it has been widely applied to $Z_{2}$ topological insulators ${ }^{22-28}$. The initial efforts to create $\mathrm{TCl} /$ superconductor heterostructures appear promising ${ }^{36}$, but achieving atomically flat interfaces in this geometry has been extremely difficult. This is in large part due to a strong intra-layer bonding of $(\mathrm{Pb}, \mathrm{Sn}) \mathrm{Se}$ and $(\mathrm{Pb}, \mathrm{Sn}) \mathrm{Te}$, which prevents their mechanical exfoliation and severely limits the choice of viable substrates for epitaxial thin film growth. Unlike van der Waals topological insulators $\mathrm{Bi}_{2}(\mathrm{Se}, \mathrm{Te})_{3}$ that can be grown on a slew of substrates nearly irrespective of the structural compatibility ${ }^{22,37}, \mathrm{TCl}$ thin films are known to be strongly susceptible to warping ${ }^{11,12,38}$. Thus, the growth of atomically flat TCls is best achieved on the substrates with a closely matched in-plane lattice structure ${ }^{39,40}$, whereas their growth on commonly used superconducting substrates, such as $\mathrm{NbSe}_{2}$ and elemental superconductors where the lattice mismatch is inevitably much larger than one percent, remains challenging. In this work, by the use of ultrathin "buffer" layers to bridge the structural mismatch, we successfully grow atomically flat SnTe thin films on top of a high- $T_{c}$ superconductor $\mathrm{Fe}(\mathrm{Te}, \mathrm{Se})$, and find the proximityinduced superconductivity at the surface of SnTe. 


\section{Results.}

\section{MBE growth of heterostructures.}

The starting point for our heterostructure growth is a bulk single crystal of superconducting $\mathrm{FeTe}_{0.55} \mathrm{Se}_{0.45}(\mathrm{Fe}(\mathrm{Te}, \mathrm{Se}))$ with $T_{c} \sim 14 \mathrm{~K}$, cleaved in ultra-high vacuum to expose a clean surface. Scanning tunneling microscopy (STM) topographs of the Fe(Te,Se) surface show a square lattice with $a_{0} \sim 3.9 \AA$ (Fig. 1b), which demonstrates that the $\mathrm{Fe}(\mathrm{Te}, \mathrm{Se})$ crystals cleave along the (001) direction. Instead of growing SnTe thin films directly on top of $\mathrm{Fe}(\mathrm{Te}, \mathrm{Se})$, we first deposit one quintuple layer (QL) of $\mathrm{Bi}_{2} \mathrm{Te}_{3}$ using molecular beam epitaxy (MBE) (Fig. 1a). This crucial layer serves to bridge the structural difference between $\mathrm{Fe}(\mathrm{Te}, \mathrm{Se})$ and $\mathrm{SnTe}$. Despite the obvious incompatibility between the in-plane lattices of $\mathrm{Fe}(\mathrm{Te}, \mathrm{Se})$ (square lattice with $a_{0} \sim 3.9 \AA$ ) and $\mathrm{Bi}_{2} \mathrm{Te}_{3}$ (hexagonal lattice with $a_{0} \sim 4.4 \AA$ ), weak van der Waals bonding at the interface allows for the epitaxial growth of $\mathrm{Bi}_{2} \mathrm{Te}_{3}{ }^{27}$. At the same time, $\mathrm{Bi}_{2} \mathrm{Te}_{3}$ can serve as nearly an ideal substrate for the growth of SnTe along the (111) direction, because the difference between the in-plane lattice constants of the two materials is only $\sim 1.7 \%{ }^{40}$, comparable to that between $\mathrm{SnTe}$ and the commonly used $\mathrm{BaF}_{2}$ substrates ${ }^{34}$. This allows us to deposit SnTe thin films of varying thicknesses on top of $1 \mathrm{QL} \mathrm{Bi}{ }_{2} \mathrm{Te}_{3}$ to successfully complete the heterostructure shown in Fig. 1a. We choose the $1 \mathrm{QL}$ thickness of the $\mathrm{Bi}_{2} \mathrm{Te}_{3}$ buffer layer ( $1 \mathrm{~nm}$ thick) to minimize the separation between SnTe and the superconducting substrate, which should in principle maximize the proximity-induced pairing correlations at the surface of SnTe ${ }^{41}$.

\section{Nanoscale structural and electronic characterization.}

STM topographs of SnTe(111) surface show a hexagonal lattice of Te atoms (Fig. 1d), qualitatively similar to those reported by previous experiments ${ }^{40}$. The topographs are also bias-dependent, appearing more inhomogeneous at higher bias (Fig. S1 ${ }^{49}$ ). Importantly, they are clearly distinct from the characteristic topographs of underlying $1 \mathrm{QL} \mathrm{Bi} 2 \mathrm{Te}_{3}$ (Fig. 1c). We further confirm the nature of the terminating layer of our heterostructure by measuring the step height in the STM topographs to be $\sim 0.4 \mathrm{~nm}$ (Fig. 1e), which equals the height of one $\mathrm{SnTe}(111)$ bilayer (BL).

We proceed to use low-temperature scanning tunneling spectroscopy to characterize the electronic properties of the film. We present data on three different SnTe films with nominal 
thicknesses of $\sim 3 \mathrm{BL}, \sim 6 \mathrm{BL}$ and $\sim 14 \mathrm{BL}$, labeled $\mathrm{A}, \mathrm{B}$ and $\mathrm{C}$, respectively. From differential conductance $(d l / d V)$ spectra acquired over a large energy range, we can estimate the top of the valence band (VBT), which is seen as a sharp upturn in conductance at negative energies (Fig. 2a). With increased SnTe film thickness, Fermi level shifts down towards the VBT, and it falls just above the VBT in the thickest film. Finite $d l / d V$ conductance above the VBT would be consistent with the existence of surface states spanning the Fermi level.

Quasiparticle interference (QPI) measurements provide further evidence supporting this scenario (Fig. 3), as the observed QPI morphology is qualitatively consistent with the expected Dirac cone structure of the SnTe(111) surface state ${ }^{42}$. The surface state of bulk SnTe(111) consists of a Dirac cone at $\Gamma$ and another one at each M point (Fig. 3(a)), with the cones at the two different $k$-space position possibly slightly offset in energy ${ }^{42}$. Given this surface state structure, there are three dominant inter-band scattering wave vectors, which are schematically depicted in Fig. 3(a). In the Fourier transforms of STM dl/dV maps in our samples, we observe diffuse signatures at the positions corresponding to all three of these scattering channels, at several different energies across the Fermi level (Fig. 3(b-d)). We note that $k_{z}$-dispersing bulk bands are typically not observed in QPI measurements ${ }^{43}$. The emergence of QPI vectors at these positions is consistent with the existence of the Dirac surface states in our samples. We also note that in the 2D limit, hybridization of the top and the bottom surface state can in principle lead to a gap opening at the Dirac point, but the topological nature of these states is expected to remain intact in a large range of thicknesses ${ }^{44}$.

Next, we look for signatures of induced superconductivity by measuring $d l / d V$ spectra over a narrow energy range near the Fermi level. Before depositing SnTe, average $d I / d V$ spectrum on top of $1 \mathrm{QL} \mathrm{Bi} \mathrm{Te}_{3} / \mathrm{Fe}(\mathrm{Te}, \mathrm{Se})$ shows a clear gap in the density of states symmetric with respect to the Fermi level, consistent with a proximity-induced superconducting gap at the surface of $\mathrm{Bi}_{2} \mathrm{Te}_{3}$ thoroughly explored in our previous work ${ }^{27} . d I / d V$ spectra acquired on the surface of SnTe(111) in samples A and B also show a prominent, symmetric spectral gap in the density of states (Fig. $2 b, c)$. Spectral gap variation at the surface of the two samples, both a comparable distance away from the bulk superconductor Fe(Te,Se), is likely due to different STM tips used, as well as the variations in Se:Te ratio and the concentration of excess interstitial Fe across different $\mathrm{Fe}(\mathrm{Te}, \mathrm{Se})$ 
substrates $^{45,46,47}$. Nevertheless, within a single heterostructure, the measured gap is spatially homogeneous over the nanoscale region measured, with little variation in the spectral shape (Fig. 2e). A much thicker sample $C$ only shows a small suppression in the density-of-states near the Fermi level (Fig. 2d). This would be an expected trend for a proximity-induced superconducting gap, where superconducting correlations decay away from the bulk superconductor ${ }^{41}$. The quick suppression of the gap away from the interface is possibly due to the relatively short-coherence length of $\mathrm{Fe}(\mathrm{Te}, \mathrm{Se})^{28,47}$.

To further investigate the gap observed on the surface of SnTe(111), we focus on a $40 \mathrm{~nm}$ square region of sample $A$, and track the average $d l / d V$ spectrum as a function of temperature (Fig. $2 f$ ). The measured gap becomes shallower with increased temperature, and ultimately disappears at $12 \mathrm{~K}$. This is comparable to the bulk $T_{c} \sim 14 \mathrm{~K}$ of the $\mathrm{Fe}(\mathrm{Te}, \mathrm{Se})$ substrate, and provides additional support for the superconducting origin of the gap. We fit the gap magnitude using a thermallybroadened BCS function (Fig. S2 ${ }^{49}$ ), which provides a good fit to the experimental data ${ }^{49}$. We find that the extracted gap magnitude as a function of temperature closely follows the BCS trend (Fig. 2g).

Lastly, we use spectroscopic imaging STM to spatially map the differential conductance at varying magnetic fields applied perpendicular to the sample surface. In type-II superconductors such as $\mathrm{Fe}(\mathrm{Te}, \mathrm{Se})$, magnetic field will penetrate the material in quantized vortices. If the surface of SnTe is indeed superconducting, we would expect to observe Abrikosov vortices as localized regions of low $d l / d V$ conductance in STM $d l / d V$ maps acquired at energies of the superconducting gap ${ }^{50}$. Fig. 4a-c shows $d l / d V$ maps acquired over the identical region of SnTe in varying magnetic field. All images show a clear vortex lattice, with the number of vortices scaling with the applied magnetic field, confirming the induced superconductivity at the surface of SnTe. Importantly, the induced gap is larger and persists to higher temperatures compared to what has been achieved in proximity experiments in $\mathrm{TCls}$ so far using low-temperature s-wave superconductors ${ }^{36}$. Interestingly, the vortex cores in superconducting SnTe exhibit not only the expected suppression of the coherence peaks in $d l / d V$ spectra, but also an intriguing peak in $d l / d V$ conductance centered at zero energy (Fig. 4d). Similar zero-bias conductance peaks have now been reported in vortices of several related systems, including a fraction of vortices measured on superconducting Fe(Te,Se) 
51, Fe impurities on the surface of $\mathrm{Fe}(\mathrm{Te}, \mathrm{Se})^{52}$ and a monolayer of $\mathrm{FeSe}^{53}$, and $\mathrm{Bi}_{2} \mathrm{Te}_{3} / \mathrm{Fe}(\mathrm{Te}, \mathrm{Se})$ heterostructures ${ }^{27,28}$ (Fig. S3 ${ }^{49}$ ).

\section{Discussion.}

We have demonstrated that $\mathrm{Bi}_{2} \mathrm{Te}_{3}$ buffer layer enables the realization of a novel heterostructure involving a topological crystalline insulator SnTe and a high- $T_{c}$ superconductor Fe(Te,Se). Temperature and magnetic field dependent scanning tunneling microscopy/spectroscopy measurements of the SnTe surface demonstrate induced superconductivity in SnTe, with the highest onset temperature and the largest energy gap in superconducting TCls to-date. The use of the same buffer layer can easily be extended to couple TCls to other unconventional superconductors or magnetic materials. Future experiments directly demonstrating spinmomentum locking and the superconducting gap in the surface states may shed light on the existence of topological superconductivity in proximitized TCls. Moreover, spin-polarized STM ${ }^{26}$ could be used to explore the potential emergence of Majorana zero modes in vortices. Overall, our experiments provide a new platform to study the interplay of crystalline symmetries, topology and superconductivity in a single material.

\section{Methods.}

$\mathrm{FeTe}_{1-\mathrm{x}} \mathrm{Se}_{\mathrm{x}}\left(\mathrm{x}^{\sim 0.45)}\right.$ bulk single crystals were grown using the self-flux method. During the growth process, RHEED pattern (obtained using a $15 \mathrm{keV}$ RHEED gun by Sentys Inc) was continuously monitored to establish the morphology of the surface. $\mathrm{Bi}_{2} \mathrm{Te}_{3}$ film was grown by co-evaporating 99.999\% pure Bismuth and 99.99\% pure Tellurium from K-cells (Sentys Inc) in Bi:Te flux ratio of 1:10. SnTe film was grown by co-evaporating 99.999\% Tin and 99.99\% Tellurium in Sn:Te 1:10 flux ratio. $\mathrm{Bi}_{2} \mathrm{Te}_{3}$ (SnTe) films were grown at the rate of 4 (2) minutes per nominally calculated QL (bilayer). Sample A was grown at $\sim 200{ }^{\circ} \mathrm{C}\left(\mathrm{Bi}_{2} \mathrm{Te}_{3}\right)$ and $\sim 300{ }^{\circ} \mathrm{C}(\mathrm{SnTe})$; sample B was grown at $\sim 250$ ${ }^{\circ} \mathrm{C}\left(\mathrm{Bi}_{2} \mathrm{Te}_{3}\right)$ and $\sim 300{ }^{\circ} \mathrm{C}(\mathrm{SnTe})$, but post-annealed to at least $320^{\circ} \mathrm{C}$ which evaporated the $\mathrm{Bi}_{2} \mathrm{Te}_{3}$ layer off (Fig. S4 ${ }^{49}$ ); sample $\mathrm{C}$ was grown at $\sim 180{ }^{\circ} \mathrm{C}$ (both $\mathrm{Bi}_{2} \mathrm{Te}_{3}$ and $\mathrm{SnTe}$ ), and post annealed for 30 minutes in vacuum at $\sim 270{ }^{\circ} \mathrm{C}$. Typical post-growth RHEED pattern of our heterostructures exhibits a streaky pattern characteristic of the layer-by layer MBE growth (Fig. S5 ${ }^{49}$ ). After the growth was completed, the heterostructure was transferred from the MBE to the STM within one 
hour, using a vacuum suitcase chamber held at $\sim 10^{-11}$ Torr base pressure, which can be directly connected to either MBE or STM chambers. Therefore, we emphasize that our material is only exposed to UHV conditions during the entire process from the start of the MBE growth to the completion of STM measurement.

STM measurements were acquired using Unisoku USM1300 STM at the base temperature of $\sim 4.5$ $\mathrm{K}$ (with the exception of temperature dependent data in Fig. 2g). STM tips used were home-made chemically etched metallic $\mathrm{Cr}$ tips. All spectroscopic measurements have been taken using a standard lock-in technique at $915 \mathrm{~Hz}$ frequency and a varying bias excitation as detailed in the figure captions. 

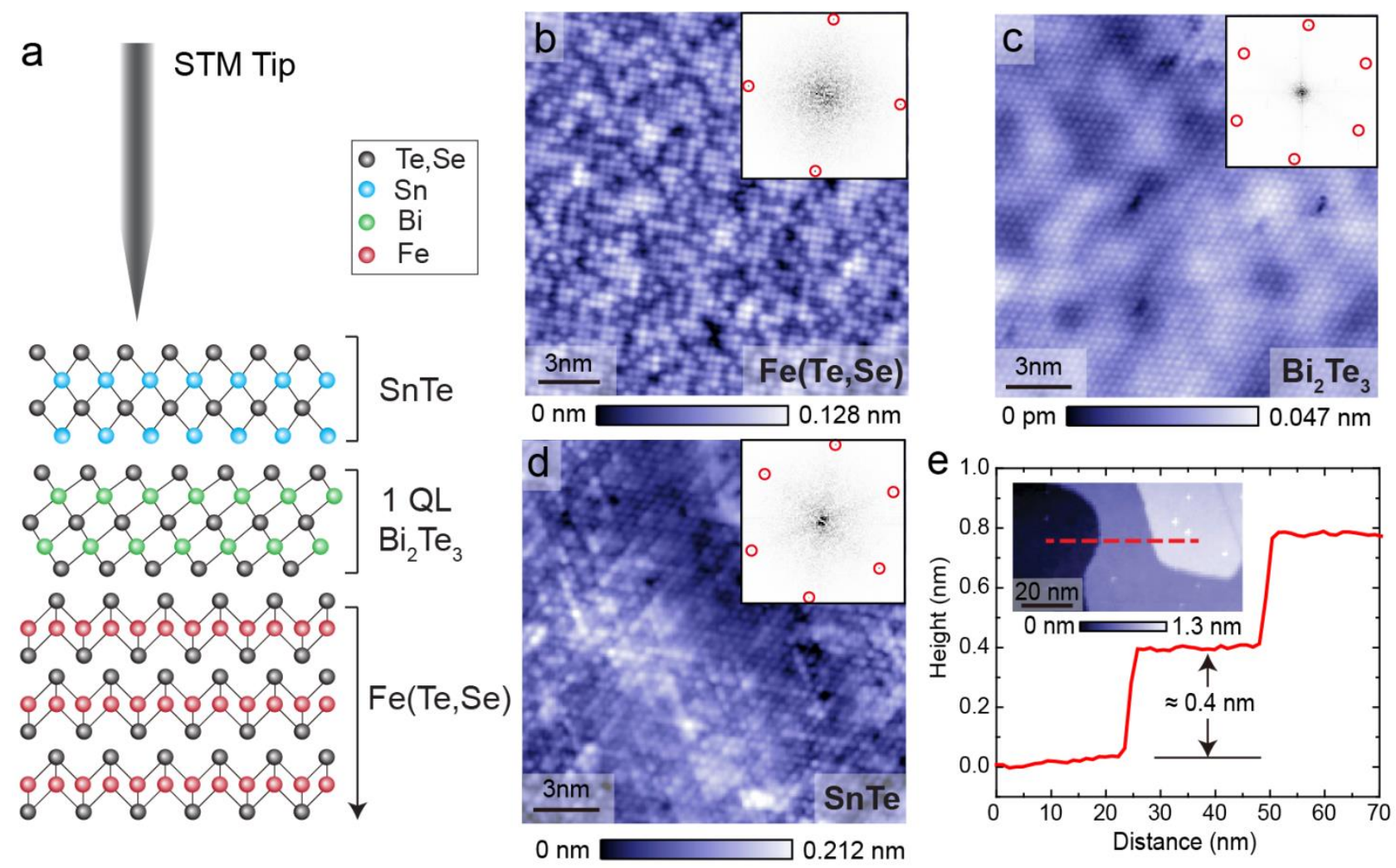

Figure 1. (a) Schematic of $\mathrm{SnTe} / \mathrm{Bi}_{2} \mathrm{Te}_{3} / \mathrm{Fe}(\mathrm{Te}, \mathrm{Se})$ heterostructure. STM topograph showing (b) exposed $\mathrm{Fe}(\mathrm{Te}, \mathrm{Se})$ substrate, (c) $1 \mathrm{QL} \mathrm{Bi}_{2} \mathrm{Te}_{3}$ buffer layer, and the (d) topmost SnTe layer. Insets in (b-d) are the respective Fourier transforms showing their lattice symmetries. (e) Height profile taken along the red dashed line in the embedded STM topograph. The step height is consistent with consecutive bilayers of SnTe. STM setup conditions: (b) $I_{\text {set }}=15 \mathrm{pA}, V_{\text {sample }}=10 \mathrm{mv}$; (c) $I_{\text {set }}=30$ $\mathrm{pA}, V_{\text {sample }}=6 \mathrm{mV}$; (d) $I_{\text {set }}=40 \mathrm{pA}, V_{\text {sample }}=40 \mathrm{mV}$; (e) $I_{\text {set }}=10 \mathrm{pA}, V_{\text {sample }}=-500 \mathrm{mV}$ 

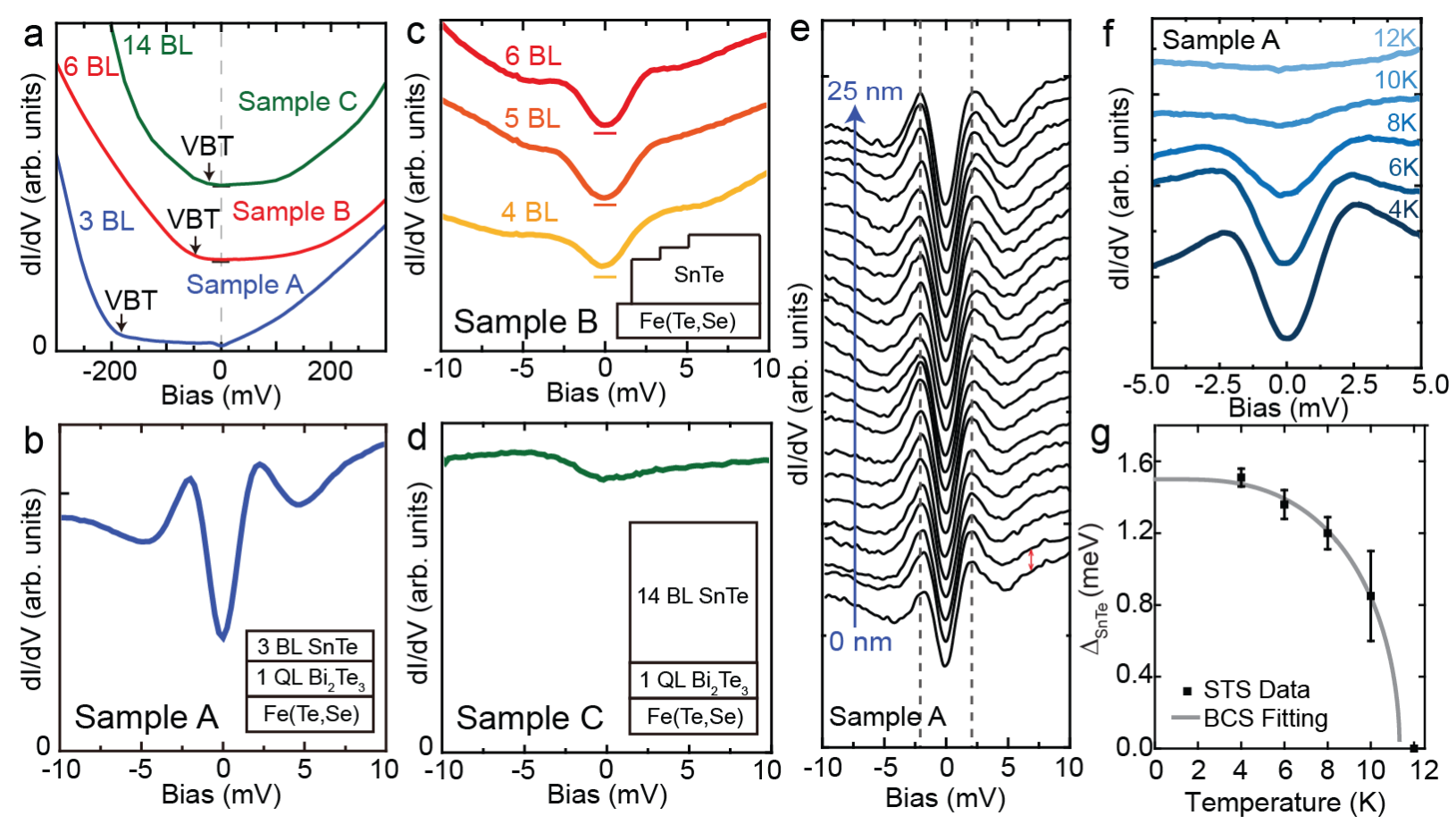

Figure 2. (a) Long range average $d l / d V$ spectra on sample $A$ (blue), sample $B$ (red) and sample $C$ (green). VBT denotes on the approximate position of the top of the valence band. (b-d) Average $d l / d V$ spectra on the surface of (b) sample A, (c) sample B and (d) sample C, with a schematic of their respective heterostructures (inset in in the lower right corner). (e) A series of $d l / d V$ spectra, offset for clarity, along a $25 \mathrm{~nm}$ line taken on the surface of sample A. (f) Average $d l / d V$ spectra, offset for clarity, as a function of temperature, showing the gap closing at $\sim 12 \mathrm{~K}$. (g) Spectral gap $\Delta_{\text {snTe }}$ as a function of temperature extracted from spectra in (f) (black squares), and the overlaid BCS trend (grey line). STM setup condition: (a) Sample A: $I_{\text {set }}=100 \mathrm{pA}, \mathrm{V}_{\text {sample }}=300 \mathrm{mV}, \mathrm{V}_{\text {exc }}=10$ $\mathrm{mV}$ (zero-to-peak); Sample B: $I_{\text {set }}=200$ pA, $V_{\text {sample }}=400 \mathrm{mV}, \mathrm{V}_{\mathrm{exc}}=10 \mathrm{mV}$; Sample C: $\mathrm{I}_{\text {set }}=100 \mathrm{pA}$, $\mathrm{V}_{\text {sample }}=300 \mathrm{mV}, \mathrm{V}_{\text {exc }}=10 \mathrm{mV}$; (b) $\mathrm{I}_{\text {set }}=40 \mathrm{pA}, \mathrm{V}_{\text {sample }}=10 \mathrm{mV}, \mathrm{V}_{\text {exc }}=0.2 \mathrm{mV}$; (c) $4 \mathrm{BL}: \mathrm{I}_{\text {set }}=100 \mathrm{pA}$, $\mathrm{V}_{\text {sample }}=10 \mathrm{mV}, \mathrm{V}_{\text {exc }}=0.2 \mathrm{mV} ; 5 \mathrm{BL}: \mathrm{I}_{\text {set }}=60 \mathrm{pA}, \mathrm{V}_{\text {sample }}=10 \mathrm{mV}, \mathrm{V}_{\mathrm{exc}}=0.2 \mathrm{mV} ; 6 \mathrm{BL}: \mathrm{I}_{\mathrm{set}}=60 \mathrm{pA}$, $\mathrm{V}_{\text {sample }}=10 \mathrm{mV}, \mathrm{V}_{\text {exc }}=0.2 \mathrm{mV}$; (d) $\mathrm{I}_{\text {set }}=150 \mathrm{pA}, \mathrm{V}_{\text {sample }}=10 \mathrm{mV}, \mathrm{V}_{\mathrm{exc}}=0.3 \mathrm{mV}$; (e) $\mathrm{I}_{\mathrm{set}}=40 \mathrm{pA}$, $\mathrm{V}_{\text {sample }}=10 \mathrm{mV}, \mathrm{V}_{\text {exc }}=0.2 \mathrm{mV}$, (f) $I_{\text {set }}=30 \mathrm{pA}, V_{\text {sample }}=5 \mathrm{mV}, V_{\text {exc }}=0.2 \mathrm{mV}$. 

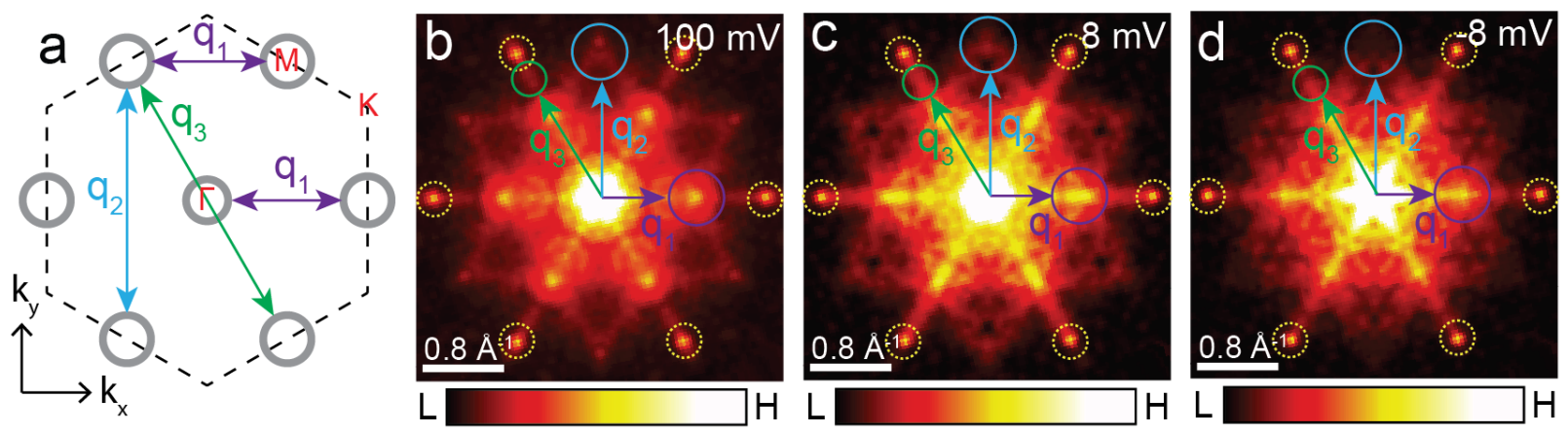

Figure 3. Quasiparticle interference (QPI) imaging of SnTe(111) surface of sample C. (a) The schematic of the SnTe (111) constant energy contour, where $q_{1}$ (purple), $q_{2}$ (blue) and $q_{3}$ (green) denote dominant scattering vectors. The dashed hexagon in (a) denotes the $1^{\text {st }}$ Brillouin zone. (bd) Fourier transforms of $\mathrm{dl} / \mathrm{dV}$ maps acquired at $100 \mathrm{mV}, 8 \mathrm{mV}$ and $-8 \mathrm{mV}$ bias, respectively. The peaks in the Fourier transform in (b) circled in purple, blue and green correspond to the scattering channels denoted in panel (a). Yellow dashed circles in (b-d) denote the atomic Bragg peaks associated with the Te lattice. STM setup conditions: (b) $I_{\text {set }}=200 \mathrm{pA}, \mathrm{V}_{\text {sample }}=100 \mathrm{mV}, \mathrm{V}_{\mathrm{exc}}=2 \mathrm{mV}$; (c) $I_{\text {set }}=200 \mathrm{pA}, V_{\text {sample }}=-8 \mathrm{mV}, V_{\text {exc }}=2 \mathrm{mV}$; (d) $I_{\text {set }}=200 \mathrm{pA}, V_{\text {sample }}=8 \mathrm{mV}, V_{\text {exc }}=2 \mathrm{mV}$. 

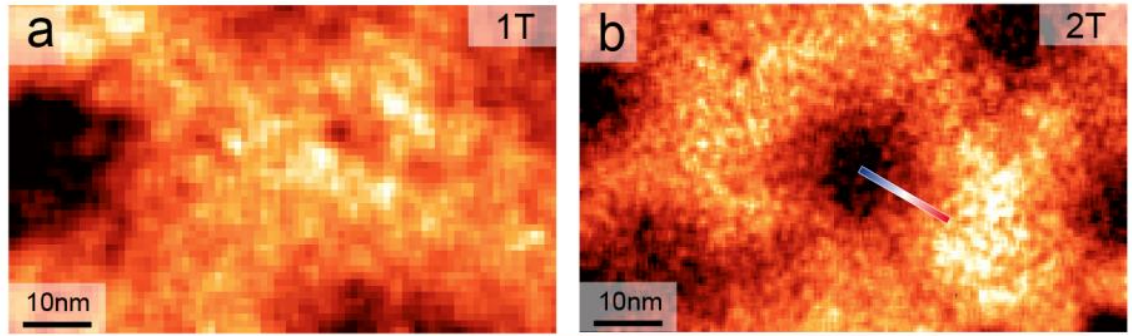

$\mathrm{L} \square \mathrm{H}$
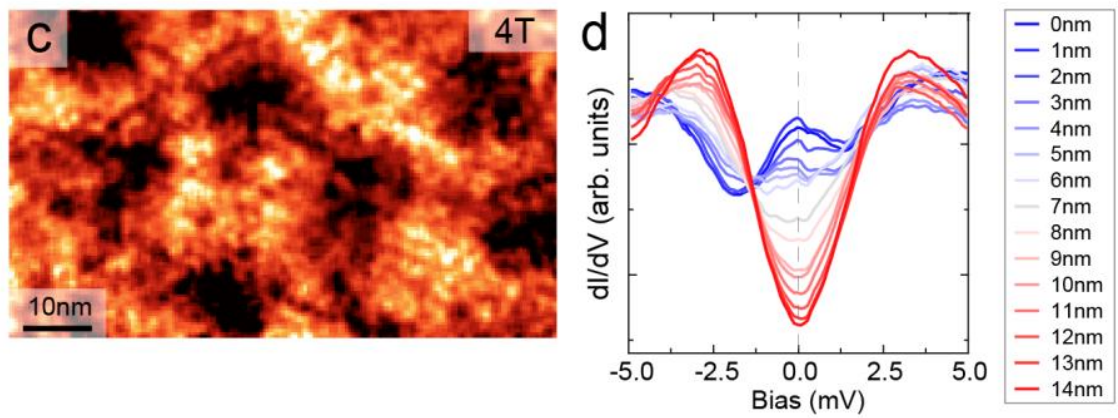

Figure 4. (a-c) $d l / d V$ maps over the same region of the sample at $1 \mathrm{~T}, 2 \mathrm{~T}$ and $4 \mathrm{~T}$ magnetic fields, respectively. All fields are applied perpendicular to the surface of the sample. (d) Radiallyaveraged $d l / d V$ spectra as a function of distance away from the vortex core in (b). Zero bias peak is visible in the vortex core (darkest blue). STM setup condition: (a) $I_{\text {set }}=4 \mathrm{pA}, V_{\text {sample }}=2 \mathrm{mV}, V_{\text {exc }}=$ $0.4 \mathrm{mV} ;(\mathrm{b}) I_{\text {set }}=8 \mathrm{pA}, V_{\text {sample }}=2 \mathrm{mV}, V_{\text {exc }}=0.4 \mathrm{mV}$. 


\section{References}

1. Fu, L. Topological Crystalline Insulators. Phys. Rev. Lett. 106, 106802 (2011).

2. Hsieh, T. H. et al. Topological crystalline insulators in the SnTe material class. Nat. Commun. 3, 982 (2012).

3. Dziawa, P. et al. Topological crystalline insulator states in $\mathrm{Pb}_{1-x} \mathrm{Sn}_{x} \mathrm{Se}$. Nat. Mater. 11, 1023-7 (2012).

4. Xu, S.-Y. et al. Observation of a topological crystalline insulator phase and topological phase transition in $\mathrm{Pb}_{1-\mathrm{x}} \mathrm{Sn}_{\mathrm{x}}$ Te. Nat. Commun. 3, 1192 (2012).

5. Tanaka, Y. et al. Experimental realization of a topological crystalline insulator in SnTe. Nat. Phys. 8, 800-803 (2012).

6. Wojek, B. M. et al. Direct observation and temperature control of the surface Dirac gap in a topological crystalline insulator. Nat. Commun. 6, 8463 (2015).

7. Tanaka, Y. et al. Tunability of the k-space location of the Dirac cones in the topological crystalline insulator $\mathrm{Pb}_{1-\mathrm{x}} \mathrm{Sn}_{\mathrm{x}}$ Te. Phys. Rev. B 87, 155105 (2013).

8. Neupane, M. et al. Saddle point singularity and topological phase diagram in a tunable topological crystalline insulator (TCI). Phys. Rev. B 92, 075131 (2015).

9. Zeljkovic, I. et al. Dirac mass generation from crystal symmetry breaking on the surfaces of topological crystalline insulators. Nat. Mater. 14, 318-24 (2015).

10. Okada, Y. et al. Observation of Dirac node formation and mass acquisition in a topological crystalline insulator. Science 341, 1496-9 (2013).

11. Zeljkovic, I. et al. Strain engineering Dirac surface states in heteroepitaxial topological crystalline insulator thin films. Nat. Nanotechnol. 10, 849-853 (2015).

12. Walkup, D. et al. Interplay of orbital effects and nanoscale strain in topological crystalline insulators. Nat. Commun. 9, 1550 (2018).

13. Ando, Y. \& Fu, L. Topological Crystalline Insulators and Topological Superconductors: From Concepts to Materials. Annu. Rev. Condens. Matter Phys. 6, 361-381 (2015). 
14. Chang, K. et al. Discovery of robust in-plane ferroelectricity in atomic-thick SnTe. Science $353,274-278$ (2016).

15. Sessi, P. et al. Robust spin-polarized midgap states at step edges of topological crystalline insulators. Science 354, 1269-1273 (2016).

16. Mazur, G. P. et al. Experimental search for the origin of zero-energy modes in topological materials. ArXiv:1709.04000.

17. Iaia, D. et al. Topological nature of step edge states on the surface of topological crystalline insulator $\mathrm{Pb}_{0.7} \mathrm{Sn}_{0.3}$ Se. ArXiv:1809.10689.

18. Zhang, F., Kane, C. L. \& Mele, E. J. Topological Mirror Superconductivity. Phys. Rev. Lett. 111, 056403 (2013).

19. Chiu, C. K., Yao, H. \& Ryu, S. Classification of topological insulators and superconductors in the presence of reflection symmetry. Phys. Rev. B - Condens. Matter Mater. Phys. 88, 1-24 (2013).

20. Fang, C., Gilbert, M. J. \& Bernevig, B. A. New Class of Topological Superconductors Protected by Magnetic Group Symmetries. Phys. Rev. Lett. 112, 106401 (2014).

21. Shiozaki, K. \& Sato, M. Topology of crystalline insulators and superconductors. Phys. Rev. $B$ 90, 165114 (2014).

22. Wang, M.-X. et al. The Coexistence of Superconductivity and Topological Order in the $\mathrm{Bi}_{2} \mathrm{Se}_{3}$ Thin Films. Science 336, 52-55 (2012).

23. Xu, J.-P. et al. Artificial Topological Superconductor by the Proximity Effect. Phys. Rev. Lett. 112, 217001 (2014).

24. Xu, S.-Y. et al. Momentum-space imaging of Cooper pairing in a half-Dirac-gas topological superconductor. Nat. Phys. 10, 943-950 (2014).

25. Xu, J.-P. et al. Experimental Detection of a Majorana Mode in the core of a Magnetic Vortex inside a Topological Insulator-Superconductor $\mathrm{Bi}_{2} \mathrm{Te}_{3} / \mathrm{NbSe}_{2}$ Heterostructure. Phys. Rev. Lett. 114, 17001 (2015). 
26. Sun, H.-H. et al. Majorana Zero Mode Detected with Spin Selective Andreev Reflection in the Vortex of a Topological Superconductor. Phys. Rev. Lett. 116, 257003 (2016).

27. Zhao, H. et al. Superconducting proximity effect in a topological insulator using $\mathrm{Fe}(\mathrm{Te}, \mathrm{Se})$. Phys. Rev. B 97, 224504 (2018).

28. Chen, M., Chen, X., Yang, H., Du, Z. \& Wen, H.-H. Superconductivity with twofold symmetry in $\mathrm{Bi}_{2} \mathrm{Te}_{3} / \mathrm{FeTe}_{0.55} \mathrm{Se}_{0.45}$ heterostructures. Sci. Adv. 4, eaat1084 (2018).

29. Hashimoto, T., Yada, K., Sato, M. \& Tanaka, Y. Surface electronic state of superconducting topological crystalline insulator. Phys. Rev. B - Condens. Matter Mater. Phys. 92, 1-8 (2015).

30. Hor, Y. S. et al. Superconductivity in $\mathrm{Cu}_{x} \mathrm{Bi}_{2} \mathrm{Se}_{3}$ and its Implications for Pairing in the Undoped Topological Insulator. Phys. Rev. Lett. 104, 57001 (2010).

31. Zhong, R. D. et al. Optimizing the superconducting transition temperature and upper critical field of $S_{1-x} \ln _{x}$ Te. Phys. Rev. B 88, 20505 (2013).

32. Zhong, R. et al. Surface-state-dominated transport in crystals of the topological crystalline insulator In-doped $\mathrm{Pb}_{1-x} \mathrm{Sn}_{\mathrm{x}}$ Te. Phys. Rev. B 91, 195321 (2015).

33. Polley, C. M. et al. Observation of surface states on heavily indium-doped SnTe(111), a superconducting topological crystalline insulator. Phys. Rev. B 93, 75132 (2016).

34. Si, W. et al. Superconducting thin films of (100) and (111) oriented indium doped topological crystalline insulator SnTe. Appl. Phys. Lett. 107, 92601 (2015).

35. De Gennes, P. G. Boundary Effects in Superconductors. Rev. Mod. Phys. 36, 225-237 (1964).

36. Klett, R. et al. Proximity-Induced Superconductivity and Quantum Interference in Topological Crystalline Insulator SnTe Thin-Film Devices. Nano Lett. 18, 1264-1268 (2018).

37. He, Q. L. et al. Two-dimensional superconductivity at the interface of a $\mathrm{Bi}_{2} \mathrm{Te}_{3} / \mathrm{FeTe}$ heterostructure. Nat. Commun. 5, 4247 (2014).

38. Springholz, G. \& Wiesauer, K. Nanoscale Dislocation Patterning in PbTe/PbSe(001) LatticeMismatched Heteroepitaxy. Phys. Rev. Lett. 88, 15507 (2001).

39. Assaf, B. A. et al. Quantum coherent transport in SnTe topological crystalline insulator thin 
films. Appl. Phys. Lett. 105, 102108 (2014).

40. Yan, C. et al. Experimental Observation of Dirac-like Surface States and Topological Phase Transition in $\mathrm{Pb}_{1-\mathrm{x}} \mathrm{Sn}_{\mathrm{x}} \mathrm{Te}(111)$ Films. Phys. Rev. Lett. 112, 186801 (2014).

41. Lee, K., Vaezi, A., Fischer, M. H. \& Kim, E.-A. Superconducting proximity effect in topological metals. Phys. Rev. B 90, 214510 (2014).

42. Tanaka, Y. et al. Two types of Dirac-cone surface states on the (111) surface of the topological crystalline insulator SnTe. Phys. Rev. B 88, 235126 (2013).

43. Zeljkovic, I. et al. Mapping the unconventional orbital texture in topological crystalline insulators. Nat. Phys. 10, 572-577 (2014).

44. Liu, J. \& Fu, L. Electrically tunable quantum spin Hall state in topological crystalline insulator thin films. Phys. Rev. B 91, 081407 (2015).

45. Mizuguchi, Y. \& Takano, Y. Review of Fe Chalcogenides as the Simplest Fe-Based Superconductor. J. Phys. Soc. Japan 79, 102001 (2010).

46. Sun, Y., Shi, Z. \& Tamegai, T. Review of annealing effects and superconductivity in $\mathrm{Fe}_{1+\mathrm{y}} \mathrm{Te}_{1-\mathrm{x}} \mathrm{Se}_{\mathrm{x}}$ superconductors. Supercond. Sci. Technol. 32, 103001 (2019).

47. Hoffman, J. E. Spectroscopic scanning tunneling microscopy insights into Fe-based superconductors. Reports Prog. Phys. 74, 124513 (2011).

48. Dynes, R. C., Garno, J. P., Hertel, G. B. \& Orlando, T. P. Tunneling Study of Superconductivity near the Metal-Insulator Transition. Phys. Rev. Lett. 53, 2437-2440 (1984).

49. See Supplemental Material at [URL will be inserted by publisher] for additional spectral fitting and structural characterization of the samples.

50. Hess, H. F., Robinson, R. B., Dynes, R. C., Valles, J. M. \& Waszczak, J. V. Scanning-TunnelingMicroscope Observation of the Abrikosov Flux Lattice and the Density of States near and inside a Fluxoid. Phys. Rev. Lett. 62, 214-216 (1989).

51. Wang, D. et al. Evidence for Majorana bound states in an iron-based superconductor. Science 362, 333-335 (2018). 
52. Yin, J.-X. et al. Observation of a robust zero-energy bound state in iron-based superconductor Fe(Te,Se). Nat. Phys. 11, 543-546 (2015).

53. Liu, C. et al. Detection of a zero energy bound state induced on high temperature superconducting one-unit-cell FeSe on $\mathrm{SrTiO}_{3}$. arXiv:1807.07259.

\section{Acknowledgements}

I.Z. gratefully acknowledges the support from Army Research Office Grant No. W911NF-17-1-0399 (MBE growth) and National Science Foundation Grant No. NSF-DMR-1654041 (STM characterization). The work in Brookhaven was supported by the Office of Science, US Department of Energy under Contract No. DE-SC0012704. J.S. and R.D.Z. were supported by the Center for Emergent Superconductivity, an Energy Frontier Research Center funded by the U.S. Department of Energy, Office of Science. 